Gergely Toldi*, Gabriella Bekő, Gabriella Kádár, Emília Mácsai, László Kovács, Barna Vásárhelyi and Attila Balog

\title{
Soluble urokinase plasminogen activator receptor (suPAR) in the assessment of inflammatory activity of rheumatoid arthritis patients in remission
}

\section{Abstract}

Background: Soluble urokinase plasminogen activator receptor (suPAR) is a biomarker increasingly used for the assessment of systemic inflammation. We aimed to evaluate suPAR for the assessment of inflammatory activity in rheumatoid arthritis (RA) patients in remission.

Methods: In our cross-sectional study we measured plasma suPAR and C-reactive protein (CRP) levels as well as erythrocyte sedimentation rate (ESR) in 120 RA patients at various stages of disease activity and 29 healthy agematched controls.

Results: suPAR, CRP and ESR values were higher in RA patients compared to healthy individuals. When SuPAR levels were analyzed according to DAS28 scores of RA patients, suPAR level in the subgroup with DAS28 $\leq 2.6$ was lower than in the subgroup with DAS28 $>2.6$, but still higher than in controls [4.45 (3.33-5.56) ng/mL vs. 3.66 (3.10-4.67) ng/mL vs. 2.80 (2.06-3.42) ng/mL, p<0.0001, median (interquartile range)]. In contrast, CRP and ESR values were comparable in the subgroup with DAS28 $\leq 2.6$ and in healthy individuals. We further analyzed the correlation between the number of tender and/or swollen joints and suPAR levels in RA patients in remission. suPAR values were significantly higher in patients with four tender and/or swollen joints than in patients with $2-3$ or $0-1$ tender and/or swollen joints.

Conclusions: While CRP and ESR values indicate remission of the chronic inflammatory process in RA, SuPAR values are still elevated compared to healthy individuals. suPAR might be particularly valuable in the recognition of inflammatory activity in patients who are in remission according to DAS28 scores but have symptoms of tender and/or swollen joints.

Keywords: C-reactive protein (CRP); erythrocyte sedimentation rate (ESR); inflammation; rheumatoid arthritis; soluble urokinase plasminogen activator receptor (suPAR).
*Corresponding author: Gergely Toldi, First Department of Pediatrics, Semmelweis University, Budapest, Bókay u. 53-54, 1083 Hungary, Phone: +36 20 4367181, Fax: +36 1 3138212,

E-mail: toldigergely@yahoo.com Barna Vásárhelyi: Research Group of Pediatrics and Nephrology, Hungarian Academy of Sciences, Budapest, Hungary Gabriella Bekő and Barna Vásárhelyi: Department of Laboratory Medicine, Semmelweis University, Budapest, Hungary Gabriella Kádár, László Kovács and Attila Balog: Department of Rheumatology, Albert Szent-Györgyi Health Center, University of Szeged, Szeged, Hungary

Emília Mácsai: Department of Internal Medicine, Csolnoky Hospital, Hungary

\section{Introduction}

Soluble urokinase plasminogen activator receptor (suPAR) is a biomarker increasingly used for the monitoring of systemic inflammation. suPAR is derived from the cleavage and release of the membrane-bound protein, urokinasetype plasminogen activator receptor ( $\mathrm{UPAR}$ ), expressed by various immune cells, trophoblasts, endothelial cells, smooth muscle cells, and by certain tumor cells [1, 2]. suPAR is detectable in low, but constant concentrations in plasma of healthy individuals [3, 4]. However, activation of the immune system and the development of an inflammatory response lead to elevated plasma suPAR concentrations. The plasma level of suPAR is increased in infectious [5, 6], autoimmune [7], neoplastic [8] and pregnancy-related [9] diseases. Interestingly, in all of these conditions suPAR concentrations are directly proportional to a worse prognosis of the disease $[5,6,8,10]$.

Rheumatoid arthritis (RA) is also characterized by a chronic inflammatory response, resulting in the erosion of the cartilage and bone, and invasive growth of synovial pannus tissue. C-reactive protein (CRP) and erythrocyte sedimentation rate (ESR) are widely used to assess the inflammatory status of RA patients, and are also used for 
the calculation of Disease Activity Score (DAS28) in RA [11]. RA is regarded as active above a DAS28 score of 2.6, however, patients in remission (DAS28 score $\leq 2.6$ ) might also be affected by inflammatory activity. Although it would have important prognostic and therapeutic consequences in the follow-up of such patients, the sensitivity of routinely used inflammatory markers does not allow early detection of this condition.

Therefore, in our investigation, we aimed to evaluate suPAR for the assessment of inflammatory activity in rheumatoid arthritis (RA) patients in remission.

\section{Materials and methods}

We enrolled 29 healthy individuals and 120 RA patients at various stages of disease duration and activity. Clinical parameters of study participants are summarized in Table 1. Healthy individuals and RA patients were age and gender matched. RA patients were diagnosed and classified according to the latest ACR/EULAR criteria [12]. All RA patients were diagnosed at least 1 year before sampling. Patients with ongoing infection or cancer were excluded from the analysis. The median of RA duration was 27 months in the patient group, and the median of DAS28 activity score was 2.8 , corresponding to low disease activity. DAS28 scores were calculated at the time of sampling. All RA patients received a variety of disease modifying antirheumatic drugs (DMARDs), containing at least one of the following components: methotrexate, leflunomid, sulfasalazine, chloroquine. Thirty-four of 120 RA patients received anti-tumor necrosis factor (TNF) therapy, while 60 of 120 patients received glucocorticoid treatment as well. Only patients with normal leukocyte count values were

\begin{tabular}{lrr} 
Characteristics & $\begin{array}{r}\text { Healthy } \\
\text { individuals } \\
\mathrm{n}=\mathbf{2 9}\end{array}$ & $\begin{array}{r}\text { RA patients } \\
\mathrm{n}=\mathbf{1 2 0}\end{array}$ \\
\hline Age, years & $55(46-69)$ & $61(48-72)$ \\
Gender, male/female & $10 / 18$ & $46 / 74$ \\
RA duration, months & - & $27(16-52)$ \\
DAS28 & - & $2.8(2.1-3.6)$ \\
Number of patients receiving & - & $34(28.3 \%)$ \\
anti-TNF therapy & - & $60(50 \%)$ \\
No. of patients receiving & & \\
glucocorticoid therapy & 2.80 & $4.24^{\mathrm{a}}$ \\
suPAR, $\mathrm{ng} / \mathrm{mL}$ & $(2.06-$ & $(3.19-5.40)$ \\
& $3.42)$ & \\
CRP, $\mathrm{mg} / \mathrm{L}$ & 2.70 & $4.00^{\mathrm{a}}$ \\
& $(\mathrm{BLD}-4.15)$ & $(\mathrm{BLD}-9.83)$ \\
ESR, $\mathrm{mm} / \mathrm{h}$ & $10(7-14)$ & $21^{\mathrm{a}}(12-36.5)$ \\
\hline
\end{tabular}

Table 1 Clinical characteristics of healthy individuals and RA patients.

Data are expressed as median (interquartile range). ${ }^{a} p<0.05$ vs. healthy individuals. included in the analysis. Healthy controls had a negative history of rheumatic symptoms and negative status upon detailed physical and laboratory examination. Written informed consent was obtained from all subjects, and our study was reviewed and approved by an independent Ethics Committee of the institution. Laboratory studies and interpretations were performed on coded samples lacking personal and diagnostic identifiers. The study was adhered to the tenets of the most recent revision of the Declaration of Helsinki.

Plasma was isolated from EDTA anticoagulated fasting blood samples and stored at $-80^{\circ} \mathrm{C}$ until measurement. Plasma suPAR concentrations were measured with the suPARnostic Flex ELISA assay (ViroGates A/S, Birkerød, Denmark). CRP levels were measured using a Roche Hitachi 912 instrument with Roche Tina-quant CRP immuno-turbidimetric assay (Roche Diagnostics GmbH, Mannheim, Germany). CRP values below the level of detection were regarded as ' 1 '. For the determination of ESR, the Westergren method was performed according to ICSH specifications [13] on undiluted EDTA anticoagulated blood samples using glass pipettes (Greiner Bio-One, Kremsmuenster, Austria). During sedimentation, the pipettes were mounted vertically on appropriate supporting racks and kept at room temperature, which never exceeded $25^{\circ} \mathrm{C}$.

Data are expressed as median (interquartile range). Comparisons between healthy individuals and RA patients were made with MannWhitney tests. In case of comparisons between healthy controls and RA patient subgroups, the Kruskal-Wallis test was performed. To test the correlation between suPAR levels and other investigated parameters, Spearman tests were used. To exclude the potential independent effect of anti-TNF and glucocorticoid therapy on the assessed factors, multiple regression analysis was used. Area under the curve (AUC) values of receiver operating characteristics (ROC) curves were calculated using standard methods and data are presented as AUC ROC ( $95 \%$ CI). p-Values $<0.05$ were considered significant. Statistics were calculated using the R software (R Development Core Team, R Foundation for Statistical Computing, Vienna, Austria).

\section{Results}

suPAR, CRP and ESR values were higher in RA patients compared to healthy individuals (Table 1). Correlation analyses revealed a correlation between suPAR values and DAS28 scores in RA patients ( $\mathrm{p}=0.02, \mathrm{r}=0.26$, Figure $1 A)$, suPAR values and ESR values in RA patients ( $p=0.05$, $r=0.30$, Figure $1 B$ ) and suPAR values and CRP values in healthy individuals $(\mathrm{p}=0.02, \mathrm{r}=0.32$, Figure $1 \mathrm{C})$.

When suPAR levels were analyzed according to different subgroups of RA patients (according to DAS28 scores, antiTNF and glucocorticoid therapy, CRP and ESR values), the levels were higher in each subgroup compared to healthy controls. When suPAR levels were compared between the corresponding subgroups, the only difference detected was between the subgroup with a DAS28 score $\leq 2.6$ and the subgroup with a DAS28 score $>2.6$ (Table 2).

CRP and ESR values were also analyzed according to subgroups of RA patients according to DAS28 scores (Table 3). Irrespectively of anti-TNF and glucocorticoid 

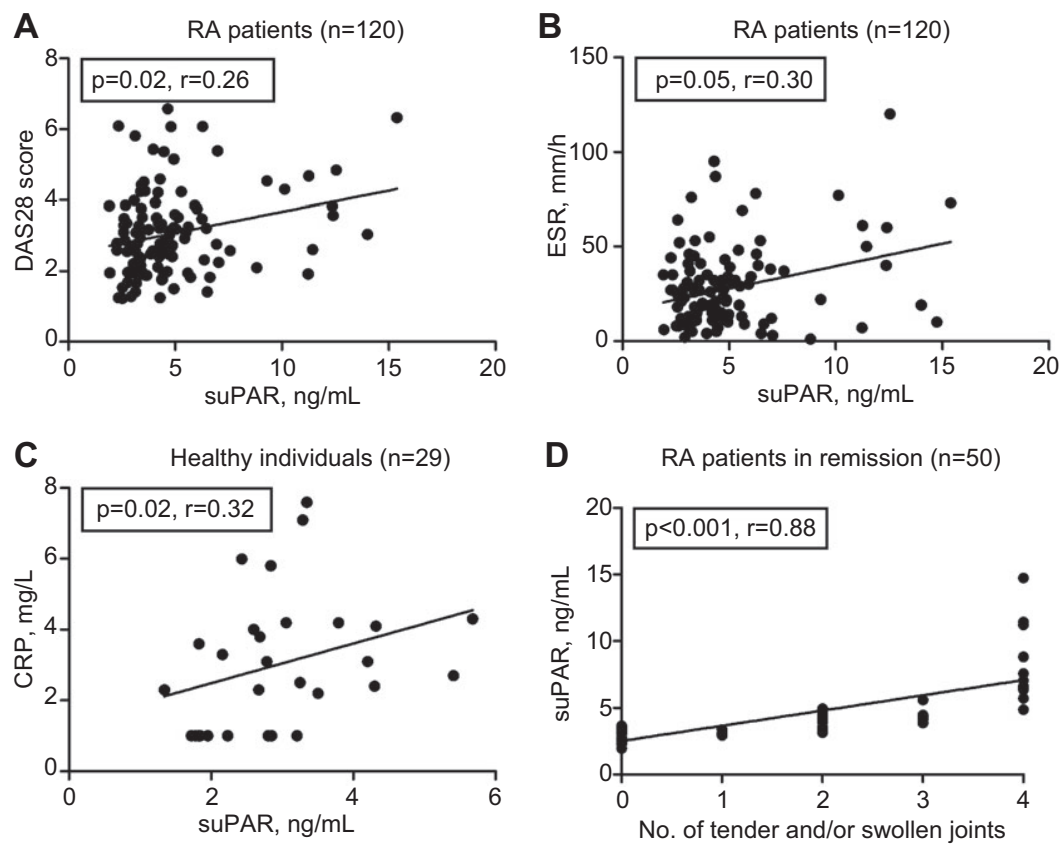

Figure 1 Correlation between suPAR plasma levels and other investigated parameters in different study groups.

(A) suPAR levels were correlated with DAS28 in all RA patients. (B) suPAR levels were correlated with ESR in all RA patients. (C) suPAR levels were correlated with CRP levels in healthy individuals. (D) suPAR levels were correlated with the number of tender and/or swollen joints in RA patients in remission.

therapy, CRP and ESR values were higher in the subgroup with a DAS28 score $>2.6$ than in RA patients in remission (DAS28 score $\leq 2.6$ ) or in healthy individuals. However, no difference was detected between patients in remission and healthy individuals.

\section{Study group}

Healthy individuals

RA patients according to DAS28 score

$\leq 2.6(41.6 \%)$

$>2.6(58.4 \%)$

RA patients according to anti-TNF therapy

Yes $(28.3 \%)$

No $(71.7 \%)$

RA patients according to glucocorticoid therapy

Yes $(50 \%)$

No $(50 \%)$

RA patients according to CRP values

$\leq 5(47.5 \%)$

$>5(52.5 \%)$

RA patients according to ESR values

$\leq 20(46.6 \%)$

$>20(53.4 \%)$

Table 2 suPAR levels in different subgroups of RA patients. Data are expressed as median (interquartile range). ${ }^{a} p<0.05$ vs. healthy individuals, ${ }^{\mathrm{b}} \mathrm{p}<0.05 \mathrm{vs}$. RA patients according to DAS 28 score $\leq 2.6$.
We further analyzed the correlation between the number of tender and/or swollen joints and suPAR levels in RA patients in remission. A strong correlation was detected $(\mathrm{p}<0.001, r=0.8$, Figure 1D). suPAR values were significantly higher in patients with four tender and/or swollen joints than in patients with $2-3$ or $0-1$ tender and/ or swollen joints. However, no difference was detected between groups of patients in remission according to the number of tender and/or swollen joints with regard to CRP and ESR values (Table 4, Figure 2).

ROC analysis of suPAR values in RA patients in remission yielded an AUC of 0.996 (95\% CI 0.984-1.007, $\mathrm{p}=0.0001$, Figure 3 ). The cut-off value of suPAR to discriminate between patients with four tender and/or swollen joints and those with 0-3 tender and/or swollen joints was $4.785 \mathrm{ng} / \mathrm{mL}$ [sensitivity\% (95\% CI) 100.0 (71.51-100.0), specificity\% (95\% CI) 95.24 (83.84-99.42)].

$4.19^{\mathrm{a}}(3.12-4.94)$

$4.27^{\mathrm{a}}(3.19-6.27)$

$4.04^{\mathrm{a}}(3.15-4.86)$

$4.44^{\mathrm{a}}(3.24-5.84)$

\section{Discussion}

The severity of RA is evaluated based on laboratory markers and clinical symptoms. Prompt classification of patients according to disease activity has significant therapeutic consequences. Therefore, it is of importance to find reliable markers that enable a more objective 


\begin{tabular}{lrrr} 
Study group & suPAR, $\mathbf{n g} / \mathbf{m L}$ & CRP, mg/L & ESR, mm/h \\
\hline Healthy individuals & $2.80(2.06-3.42)$ & $2.70($ BLD-4.15) & $10(7-14)$ \\
RA patients according to DAS28 score & & & $13(9-18)$ \\
$\leq 2.6(41.6 \%)$ & $3.66^{\mathrm{a}}(3.10-4.67)$ & $2.25(\mathrm{BLD}-5.28)$ & $32^{\mathrm{a}, \mathrm{b}}(20-47)$ \\
$>2.6(58.4 \%)$ & $4.45^{\mathrm{a}, \mathrm{b}}(3.33-5.56)$ & $6.18^{\mathrm{a}, \mathrm{b}}(2.50-11.48)$ & \\
\hline
\end{tabular}

Table 3 Inflammatory parameters in RA patients according to DAS28. Data are expressed as median (interquartile range). ${ }^{\mathrm{a}} \mathrm{p}<0.05 \mathrm{vs}$. healthy individuals, ${ }^{\mathrm{b}} \mathrm{p}<0.05 \mathrm{vs}$. RA patients according to DAS 28 score $\leq 2.6$.

\begin{tabular}{lcrr} 
Study group & suPAR, $\mathbf{n g} / \mathbf{m L}$ & CRP, $\mathbf{m g} / \mathbf{L}$ & ESR, $\mathbf{m m} / \mathbf{h}$ \\
\hline Healthy individuals & $2.80(2.06-3.42)$ & $2.70(B L D-4.15)$ & $10(7-14)$ \\
RA patients in remission & \\
$0-1(45.3 \%)$ & $3.04(2.60-3.32)$ & $2.50(B L D-5.73)$ & $12(8-18)$ \\
$2-3(34.0 \%)$ & $4.33^{\mathrm{a}, \mathrm{b}}(3.93-4.50)$ & $2.70(\mathrm{BLD}-5.43)$ & $17(12-18)$ \\
$4(20.7 \%)$ & $7.04^{\mathrm{a}, \mathrm{b}, \mathrm{c}}(6.37-11.23)$ & $2.60(\mathrm{BLD}-10.40)$ & $9(4-37)$ \\
\hline
\end{tabular}

Table 4 Inflammatory parameters in RA patients in remission.

Data are expressed as median (interquartile range). ${ }^{a} p<0.05 \mathrm{vs}$. healthy individuals, ${ }^{\mathrm{b}} \mathrm{p}<0.05 \mathrm{vs}$. RA patients with $0-1$ tender and/or swollen joints, ${ }^{c} p<0.05$ vs. RA patients with $2-3$ tender and/or swollen joints.

evaluation than the presence or lack of clinical symptoms, especially in the follow-up of patients in remission with inflammatory activity. Based on previous studies, suPAR is a sensitive marker of inflammation.

In our study, suPAR levels as well as CRP and ESR values were elevated in a group of RA patients with various durations and activity of disease compared to healthy controls (Table 1). This is in agreement with earlier results of Slot et al. who also found higher plasma suPAR concentrations in RA. Furthermore, in their study suPAR levels in RA were positively correlated to CRP and ESR values. They concluded that increased suPAR levels might reflect erosive activity in RA [14]. It is of interest that we also found a correlation between ESR and DAS28 but not CRP values and suPAR levels investigating all RA patients (Figure 1A, B). A reason for this finding might be that in our study, ESR was used for the calculation of DAS28 scores, therefore, alterations of DAS28 are related to ESR values. Furthermore, the patients enrolled in our study were more heterogenous in terms of disease severity, including patients with milder RA. In contrast with the findings in RA, CRP values were correlated to suPAR levels in healthy individuals in our study (Figure 1C).

Next we investigated suPAR levels in different subgroups of RA patients. When RA patients were grouped
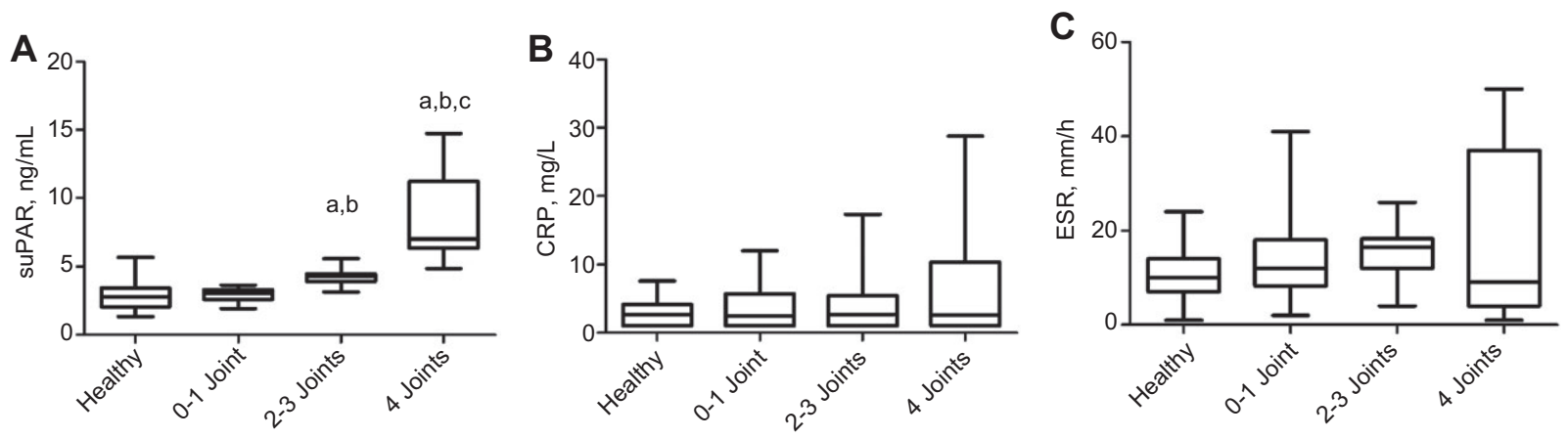

Figure 2 Comparison of suPAR plasma levels (A), CRP plasma levels (B) and ESR (C) in healthy individuals and RA patients in remission grouped according to the number of tender and/or swollen joints.

Horizontal line: median, box: interquartile range, whisker: range. ${ }^{\mathrm{a}} \mathrm{p}<0.05 \mathrm{vs}$. healthy individuals, ${ }^{\mathrm{b}} \mathrm{p}<0.05$ vs. RA patients with $0-1$ tender and/or swollen joints, ${ }^{c} p<0.05$ vs. RA patients with $2-3$ tender and/or swollen joints. 


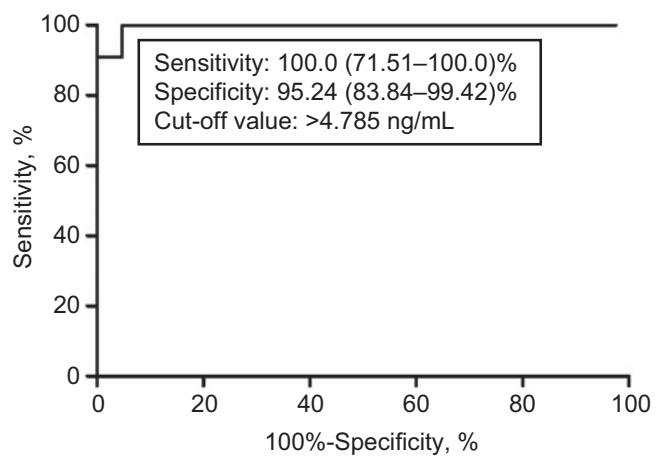

Figure 3 ROC analysis of suPAR values in RA patients in remission. Patients with four tender and/or swollen joints were regarded as patients under risk for clinical relapse. AUC: $0.996(95 \% \mathrm{CI}$ 0.984-1.007, $p=0.0001$ ).

according to anti-TNF and glucocorticoid therapy, or CRP and ESR values, no differences were detected between the corresponding subgroups. However, when we compared RA subgroups according to DAS28 scores, a difference between RA patients in remission (DAS28 $\leq 2.6$ ) and patients with different stages of active disease (DAS28 > 2.6) was noted (Table 2).

Recent studies demonstrated that suPAR has many advantages over CRP and ESR in the detection of lowgrade inflammation. In contrast with these markers, suPAR levels in healthy individuals are stable throughout the day, independently whether the subject is fasting or not [15]. Furthermore, even repeated freeze-thaw procedures of plasma samples do not affect suPAR concentrations [16]. Our results also suggest that suPAR is a more sensitive marker of inflammatory activity in RA patients in remission according to DAS28 scores. While CRP and ESR values were comparable with healthy individuals in this group of patients, suPAR values were elevated. However, suPAR levels in patients in remission were lower than in patients with DAS28 > 2.6. In contrast, CRP and ESR values were comparable in patients in remission and in healthy controls (Table 3).

Remission is defined as DAS28 $\leq 2.6$. However, it has to be noted that if both ESR and visual analogue scale (VAS) values are low upon DAS28 assessment, the presence of a maximum of four tender and/or swollen joints still results in a DAS28 score corresponding to remission. Therefore, we investigated the presence of clinical symptoms in RA patients in remission, and further grouped them according to the number of tender and/or swollen joints. The number of affected joints was strongly correlated to elevated plasma suPAR levels, indicating that suPAR levels represent well the presence of ongoing inflammatory activity in RA patients in remission (Figure
1D). Interestingly, higher levels of suPAR were associated with worse prognosis in several other diseases, including infections $[5,6]$ and tumors $[8,10]$. While CRP and ESR values were similar in all subgroups of RA patients in remission to the levels seen in healthy individuals, suPAR values were elevated indicating the inflammatory activity in patients with 2-3 or four affected joints. Highest suPAR values were observed in patients with the highest number of affected joints. Of note, this subgroup (11 patients) gave almost 10\% of the whole RA group of our study and over $20 \%$ of RA patients in remission, indicating that determination of the inflammatory activity in RA patients in remission based on plasma suPAR values might be regularly needed during follow-up. ROC analysis (Figure 3 ) revealed a suPAR cut-off value of 4.785 $\mathrm{ng} / \mathrm{mL}$ to identify patients under risk for clinical relapse (i.e., patients with four tender and/or swollen joints in contrast with patients with $0-3$ tender and/or swollen joints). Moreover, recent data also indicated that patients in remission according to DAS28 scores could also have slowly progressive structural damage without relevant clinical symptoms and with normal CRP and ESR [17]. In such cases only ultrasound investigation of the joints supports the presence of synovitis. However, the use of ultrasound has limited efficiency in detecting inflammation, since its availability is restricted, it is time-consuming, and investigator-dependent.

In summary, suPAR is a sensitive and stable marker of inflammation in the monitoring of RA patients. Based on plasma suPAR levels, we have identified a subgroup of RA patients in remission according to DAS28 scores, who have clinical symptoms of inflammatory activity (tender and/or swollen joints) which correlate to elevated plasma suPAR levels. Importantly, ESR and CRP values showed no alteration in these patients compared to healthy controls. Hence, suPAR might be particularly valuable in the recognition of inflammatory activity in patients who are in remission according to DAS28 scores but have clinical symptoms affecting joints. Monitoring of suPAR levels might be a very helpful tool for the follow-up of patients in remission with mild clinical signs. Our finding might also have important therapeutic consequences, since this group of patients identified by elevated SUPAR levels may receive earlier treatment of the inflammatory activity.

Acknowledgments: This work was supported by the Hungarian Scientific Research Fund [OTKA; grant number 101661]; the Medical Research Council [grant number ETT 05-180/2009]; and a research grant from Roche Hungary Ltd. 


\section{Conflict of interest statement}

Authors' conflict of interest disclosure: The authors stated that there are no conflicts of interest regarding the publication of this article. Research funding played no role in the study design; in the collection, analysis, and interpretation of data; in the writing of the report; or in the decision to submit the report for publication.

\section{References}

1. Danø K, Behrendt N, Brünner N, Ellis V, Ploug M, Pyke C. The urokinase receptor. Protein structure and role in plasminogen activation and cancer invasion. Fibrinolysis 1994;8:189-203.

2. Behrendt N, Stephens RW. The urokinase receptor. Fibrinolysis Proteol 1998;12:191-204.

3. Stephens RW, Pedersen AN, Nielsen HJ, Hamers MJ, HøyerHansen G, Rønne E, et al. ELISA determination of soluble urokinase receptor in blood from healthy donors and cancer patients. Clin Chem 1997;43:1876-84.

4. Rønne E, Pappot H, Grøndahl-Hansen J, Høyer-Hansen G, Plesner $\mathrm{T}$, Hansen NE, et al. The receptor for urokinase plasminogen activator is present in plasma from healthy donors and elevated in patients with paroxysmal nocturnal haemoglobinuria. $\mathrm{Br}$ J Haematol 1995;89:576-81.

5. Ostergaard C, Benfield T, Lundgren JD, Eugen-Olsen J. Soluble urokinase receptor is elevated in cerebrospinal fluid from patients with purulent meningitis and is associated with fatal outcome. Scand J Infect Dis 2004;36:14-9.

6. Ostrowski SR, Ullum H, Goka BQ, Høyer-Hansen G, Obeng Adjei G, Pedersen BK, et al. Plasma concentrations of soluble urokinase-type plasminogen activator receptor are increased in patients with malaria and are associated with a poor clinical or a fatal outcome. J Infect Dis 2005;191:1331-41.

7. Balabanov R, Lisak D, Beaumont T, Lisak RP, Dore-Duffy P. Expression of urokinase plasminogen activator receptor on monocytes from patients with relapsing-remitting multiple sclerosis: effect of glatiramer acetate (copolymer 1). Clin Diagn Lab Immunol 2001;8:1196-203.

8. Sier CF, Stephens R, Bizik J, Mariani A, Bassan M, Pedersen N, et al. The level of urokinase-type plasminogen activator receptor is increased in serum of ovarian cancer patients. Cancer Res 1998;58:1843-9.

9. Toldi G, Bíró E, Szalay B, Stenczer B, Molvarec A, Rigó J Jr, et al. Soluble urokinase Plasminogen Activator Receptor (suPAR)
Research funding: None declared.

Employment or leadership: None declared.

Honorarium: None declared.

Received April 4, 2012; accepted May 20, 2012; previously published online June 21, 2012 levels in healthy pregnancy and preeclampsia. Clin Chem Lab Med 2011;49:1873-6.

10. Thunø M, Macho B, Eugen-Olsen J. suPAR: the molecular crystal ball. Dis Markers 2009;27:157-72.

11. Wells G, Becker JC, Teng J, Dougados M, Schiff M, Smolen J, et al. Validation of the 28-joint Disease Activity Score (DAS28) and European League Against Rheumatism response criteria based on C-reactive protein against disease progression in patients with rheumatoid arthritis, and comparison with the DAS28 based on erythrocyte sedimentation rate. Ann Rheum Dis 2009;68:954-60.

12. Aletaha D, Neogi T, Silman AJ, Funovits J, Felson DT, Bingham CO 3rd, et al. 2010 Rheumatoid arthritis classification criteria: an American College of Rheumatology/European League Against Rheumatism collaborative initiative. Arthritis Rheum 2010;62:2569-81.

13. [No authors listed] ICSH recommendations for measurement of erythrocyte sedimentation rate. International Council for Standardization in Haematology (Expert Panel on Blood Rheology) J Clin Pathol 1993;46:198-203.

14. Slot O, Brünner N, Locht H, Oxholm P, Stephens RW. Soluble urokinase plasminogen activator receptor in plasma of patients with inflammatory rheumatic disorders: increased concentrations in rheumatoid arthritis. Ann Rheum Dis 1999;58:488-92.

15. Sier CF, Sidenius N, Mariani A, Aletti G, Agape V, Ferrari A, et al. Presence of urokinase-type plasminogen activator receptor in urine of cancer patients and its possible clinical relevance. Lab Invest 1999;79:717-22.

16. Riisbro R, Christensen IJ, Høgdall C, Brünner N, Høgdall E. Soluble urokinase plasminogen activator receptor measurements: influence of sample handling. Int J Biol Markers 2001;16:233-9.

17. Aletaha D, Smolen JS. Joint damage in rheumatoid arthritis progresses in remission according to the disease activity score in 28 joints and is driven by residual swollen joints. Arthritis Rheum 2011;63:3702-11. 